\title{
Structural Brain Network Reorganization in Patients with Neuropsychiatric Systemic Lupus Erythematosus
}

\author{
(D)X. Xu, DE.S. Hui, DM.Y. Mok, DJ. Jian, (D) C.S. Lau, and DH.K.F. Mak
}

\begin{abstract}
BACKGROUND AND PURPOSE: Patients with neuropsychiatric systemic lupus erythematosus have worse outcomes compared with those with systemic lupus erythematosus. A better understanding of the mechanisms of neuropsychiatric systemic lupus erythematosus could potentially improve diagnosis and management. The goal of this study was to investigate the differences in the structural brain network of patients with neuropsychiatric systemic lupus erythematosus compared with patients with systemic lupus erythematosus by using brain connectivity analysis.
\end{abstract}

MATERIALS AND METHODS: We recruited 20 subjects for each patient cohort and age-matched healthy controls. The topology and efficiency of the network and the characteristics of various brain hubs were investigated by using brain connectivity analysis of diffusion MR imaging data.

RESULTS: There were more extensive reorganizations in the structural brain network of patients with neuropsychiatric systemic lupus erythematosus than in patients with systemic lupus erythematosus. For example, the network of the former had significantly decreased clustering coefficient and local efficiency. They also had significantly lower nodal efficiency in the superior temporal gyrus $(P=.046)$ and middle temporal gyrus $(P=.041)$.

CONCLUSIONS: Our results hint at a plausible relationship between the neuropsychiatric symptoms and reorganization of the structural brain network of patients with systemic lupus erythematosus. Brain connectivity analysis may be a potential tool to subtype these patients.

ABBREVIATIONS: NPSLE = neuropsychiatric systemic lupus erythematosus; SLE = systemic lupus erythematosus

$\mathbf{P}$ atients with systemic lupus erythematosus (SLE) with neuropsychiatric manifestations, known as neuropsychiatric SLE (NPSLE), have significantly worse outcomes than those without these manifestations. ${ }^{1}$ The anti-dsDNA antibody is a hallmark serologic biomarker for the definitive diagnosis of SLE. The diagnosis of NPSLE is, on the contrary, challenging and depends largely on the clinical assessment and the appropriate selection of diagnostic investigations. ${ }^{2}$ Gross radiologic abnormalities on

Received February 29, 2016; accepted after revision July 28.

From the Department of Diagnostic Radiology (X.X., E.S.H., H.K.F.M.) and Division of Rheumatology and Clinical Immunology (C.S.L.), Department of Medicine, University of Hong Kong, Pokfulam, Hong Kong Special Administrative Region; Department of Biomedical Sciences (M.Y.M.), City University of Hong Kong, Kowloon Tong, Hong Kong Special Administrative Region; and Radiology Department (J.J.), The First Affiliated Hospital, Nanchang University, Nanchang, Jiangxi China.

This work was supported by the University of Hong Kong Small Project Funding, 200907176197

Please address correspondence to Edward S. Hui, PhD, Department of Diagnostic Radiology, University of Hong Kong, Pokfulam, HKSAR; e-mail: edward.s.hui@gmail.com; Henry K.F. Mak, MD, Department of Diagnostic Radiology, University of Hong Kong, Pokfulam, HKSAR; e-mail: makkf@hku.hk

http://dx.doi.org/10.3174/ajnr.A4947 conventional MR imaging are either not often found ${ }^{3}$ or are not related to neuropsychiatric sequelae. ${ }^{4}$ Nonetheless, recent studies by using diffusion tensor imaging have demonstrated differences in the microstructures of the white matter of patients with NPSLE compared with those with SLE. ${ }^{5,6}$ That the global disease burdens of NPSLE are not well explained by focal brain abnormalities and that brain microstructural alterations may be at play suggest that changes in the architecture of brain wiring, known as the brain network, ${ }^{7}$ may likely underpin the neuropsychiatric deficits. ${ }^{8}$

To better understand and comprehensively characterize a brain network, brain connectivity analysis has been devised. ${ }^{9}$ With such analysis, it has been found that the topology of the normal human brain closely resembles that of a "small-world" network, one that is critical for normal brain functions, and optimizes the cost of information processing in the brain. ${ }^{7}$ Apart from network topology, the relationship between individual brain regions and the rest of the network can also be investigated by using brain connectivity analysis. For instance, the hubs of a brain network, the regions that have the largest number of connections with the rest of the brain, can be identified. ${ }^{10}$ The vital roles of 


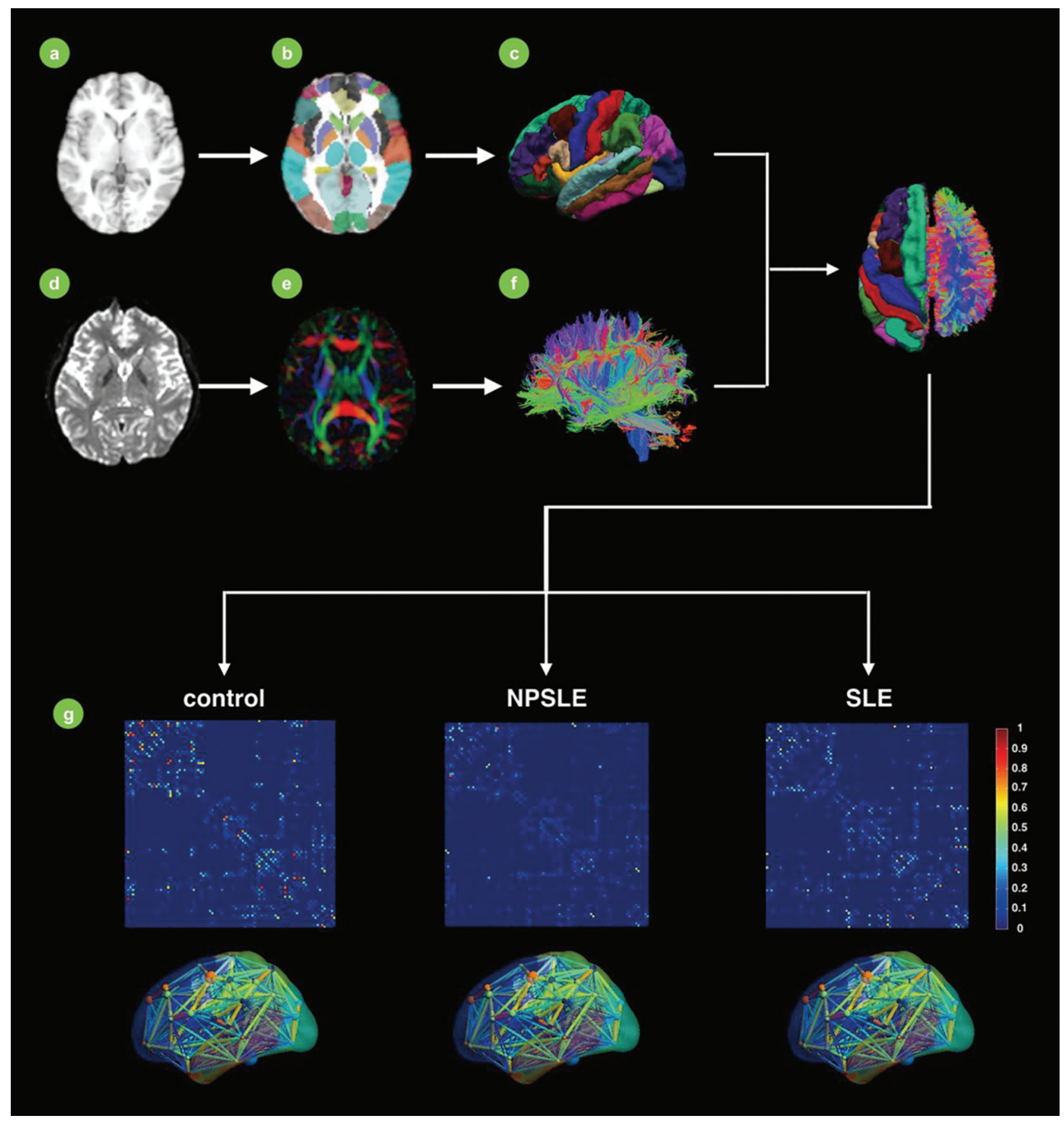

FIG 1. A flowchart of the postprocessing required for brain connectivity analysis. For each subject, T1-weighted anatomic images $(A)$ were registered to non-diffusion-weighted images and subsequently to the International Consortium for Brain Mapping 152 template. The resulting transformation matrix was then used to bring various brain regions or ROIs ( $B$ and $C$ ) from the Automated Anatomical Labeling atlas into the native anatomic image space. Diffusion tensor was obtained from diffusion-weighted images $(D)$; and its associated diffusion metrics, such as color-coded fractional anisotropy maps (red, left to right; green, anterior to posterior; blue, inferior to superior), were subsequently obtained for constructing the whole-brain white matter tractogram $(F)$. After we combined the tractogram $(F)$ and ROIs $(C)$, the connectivity matrix $(G)$, which records the connections among all ROls, was obtained for subsequent group analysis.

these hubs have been supported by studies that have demonstrated associations between the change in the properties of brain hubs and behavioral and cognitive deficits for various neuropsychiatric diseases and disorders. ${ }^{11,12}$

Considering that the diagnosis of NPSLE is far from definitive, it is imperative that a reliable neuroimaging method for subtyping patients with SLE be made available. We hypothesized that brain connectivity analysis could potentially fill this void. The goal of this study was to investigate the differences in the structural brain networks of patients with NPSLE compared with those with SLE.

\section{MATERIALS AND METHODS}

\section{Participants}

This retrospective study was approved by the Institutional Review Board of the University of Hong Kong and informed consent was obtained. The inclusion criteria were the following: patients with 
SLE who satisfied the revised American College of Rheumatology classification criteria $^{13}$; and patients with NPSLE who fulfilled the case definition of the American College of Rheumatology for neuropsychiatric lupus, ${ }^{14}$ with $\geq 1$ of the following features: serologic activities, abnormal CSF findings with the exclusion of infection, and abnormal white matter lesions on MR imaging of the brain. Subjects who had obvious dementia and a recent operation were excluded. Patients with SLE and NPSLE were matched by age, sex, and disease duration. Twenty female patients with chronic NPSLE and 20 female patients with SLE were referred from the Rheumatology Clinic of University Hospital, while 20 age-matched healthy female controls without any neurologic or psychological conditions or physical disabilities were recruited.

\section{Image Acquisition}

All scans were performed on a 3T MR imaging scanner (Achieva; Philips Healthcare, Best, the Netherlands) with an 8-channel sensitivity encoding head coil for reception. Diffusion MR imaging with non-diffusion-weighted image $\left(\mathrm{B}_{0}\right)$ with 4 averaging and diffusion-weighted images along 15 gradient directions with bvalues of $1000 \mathrm{~s} / \mathrm{mm}^{2}$ with 2 averaging were acquired by using a single-shot echo-planar imaging sequence with the following parameters: TR/TE $=9150 / 65 \mathrm{~ms}$, FOV $=225 \times 225 \mathrm{~mm}^{2}$, reconstruction resolution $=2 \times 2 \mathrm{~mm}^{2}$, section thickness $=2 \mathrm{~mm}$ (no gap), 70 sections, sensitivity encoding factor $=2$, scan time $=9$ minutes 39 seconds. For anatomic reference, T1-weighted images were acquired by using a 3D-MPRAGE sequence with the following parameters: $\mathrm{TR} / \mathrm{TE} / \mathrm{TI}=15 / 3.5 / 800 \mathrm{~ms}$, reconstruction resolution $=1 \times 1 \times 1.5 \mathrm{~mm}^{3}$, 100 sections, scan time $=6$ minutes 1 second. Axial T2-weighted images with the same geometry as that of the DTI acquisition were obtained by using a multishot turbo spin-echo sequence $(\mathrm{TR} / \mathrm{TE}=3000 / 80 \mathrm{~ms}$, reconstruction resolution $=0.33 \times 0.33 \mathrm{~mm}^{2}$, section thickness $=3 \mathrm{~mm}, 24$ sections, total scan time $=1$ minute 18 seconds).

\section{Image Processing and Structural Brain Network Construction}

The presence of radiologic abnormalities such as lacunar infarct, chronic infarct, and microbleeds were assessed from T2-weighted images by an experienced radiologist. Figure 1 summarizes the postprocessing required for performing brain-connectivity analysis by using diffusion MR imaging data.

Brain Parcellation. An Automated Anatomical Labeling atlas (http:// www.gin.cnrs.fr/AAL-216) was used to parcellate the brain into 90 cortical and subcortical regions. ${ }^{15}$ T1-weighted images were first registered to DTIs by using the FMRIB Linear Image Registration Tool (FLIRT; http://www.fmrib.ox.ac.uk). ${ }^{16}$ The native space structural images were subsequently registered to the International Consortium for Brain Mapping 152 template (http://www. bic.mni.mcgill.ca/ServicesAtlases/ICBM152NLin2009) by using the FMRIB Nonlinear Registration Tool (FNIRT; http://fsl. fmrib.ox.ac.uk/fsl/fslwiki/FNIRT). ${ }^{16}$ The inverse of the transformation matrix was subsequently applied to the atlas, thereby bringing all brain ROIs from the Automated Anatomical Labeling atlas into each subject's native structural MR imaging space. Volumetric analysis was subsequently performed by using the UCLA
Multimodal Connectivity Package (http://www.ccn.ucla.edu/ wiki/index.php/UCLA_Multimodal_Connectivity_Package). ${ }^{17}$

Diffusion MR Imaging Tractography. All DWI was first registered to $\mathrm{B}_{0}$ images to correct for eddy current distortion and head motion with the FMRIB Diffusion Toolbox (http://fsl.fmrib.ox. ac.uk/fsl/fslwiki/FDT). ${ }^{18}$ The diffusion tensor and its associated eigenvectors and eigenvalues were obtained with the Diffusion Toolbox. ${ }^{19}$ To construct the structural connections among 90 brain regions, we performed DTI-based tractography to track WM fiber tracts by using TrackVis (http://trackvis.org) with a fractional anisotropy threshold of 0.2 and a fiber turning angle threshold of $45^{\circ}$.

Brain Network. The connections among all brain regions were computed from the WM tractogram by using the UCLA Multimodal Connectivity Package. ${ }^{17}$ The structural connection was estimated by counting the number of WM fiber tracts that originate from 1 region and terminate in another. The fiber count was considered the weight of each edge. After we repeated this step for all regions, an interregional undirected weighted network with weighted connections was constructed.

\section{Brain Connectivity Analysis}

The individual's weighted connectivity matrix was normalized to its largest entry to minimize the overall differences in connectivity strength within each subject. Then for each normalized connectivity matrix, measures of small-world properties and network efficiency with the characteristics of each node were computed with the Brain Connectivity Toolbox (http://www.brain-connectivity-toolbox.net/). ${ }^{20}$

Small-World Properties. Measures of the small-world network were first introduced by Watts and Strogatz. ${ }^{21}$ The clustering coefficient of a node refers to how the neighboring nodes and the node itself are connected. The clustering coefficient of the entire network is the average of that of all nodes in the network. The characteristic shortest path length of a node refers to the average shortest travel distance between another node and the node itself. The characteristic shortest path length of the entire network is the average of that of all nodes. The clustering coefficient and characteristic shortest path length of the network are often normalized to those of 100 random networks with a preserved edge number and probability distribution of degree, respectively, to determine how a network is different from a small-world network. A network is considered small-world if the normalized clustering coefficient $(\gamma)$ is much larger than 1 and the normalized characteristic shortest path length $(\lambda)$ is close to $1 .^{21}$

Network Efficiencies. The global efficiency of a network is the average of the inverse of the shortest path length of all node pairs in the network ${ }^{22}$ and usually reflects the ability of the network in parallel information processing. The local efficiency of an entire network is the average of the global efficiency of the community neighboring all nodes in the network and is often considered an indicator of the fault tolerance of a network. ${ }^{23}$ 
Table 1: Demographics

\begin{tabular}{lcccc}
\hline & Control & NPSLE & SLE & $P$ Value \\
\hline Sample size & 20 & 19 & 19 & - \\
Age (yr) & $40.1 \pm 14.6$ & $44.2 \pm 13.4$ & $41.9 \pm 14.1$ & .822 \\
Disease duration (yr) & - & $18.0 \pm 10.1$ & $17.4 \pm 9.3$ & .954 \\
Cumulative prednisolone dosage (g) & - & $53.8 \pm 39.8$ & $48.9 \pm 28.9$ & .317 \\
Radiologic abnormalities (prevalence) (\%) & & & & \\
$\quad$ Lacunar infarct & - & 21 & 11 & .179 \\
$\quad$ Chronic infarct & - & 16 & 11 & .350 \\
$\quad$ Microbleeds & - & 11 & 11 & 1.000 \\
Neuropsychiatric symptoms (prevalence) (\%) & - & & & \\
$\quad$ Seizure & - & 53 & - & - \\
$\quad$ Confusion & - & 21 & - & - \\
$\quad$ Stroke & - & 16 & - & - \\
$\quad$ Psychosis & - & 11 & - & - \\
$\quad$ Cognitive impairment & - & 5 & - & - \\
\hline Note:--indicates not applicable & & & &
\end{tabular}

Table 2: Volumetric analysis

\begin{tabular}{lcc}
\hline & \multicolumn{2}{c}{$P$ Value } \\
\cline { 2 - 3 } \multicolumn{1}{c}{ Brain Regions } & NPSLE vs Controls & SLE vs Controls \\
\hline L. Precentral gyrus & $.016^{\mathrm{a}}$ & $.007^{\mathrm{b}}$ \\
L. Middle frontal gyrus & $.005^{\mathrm{b}}$ & $.001^{\mathrm{b}}$ \\
R. Middle frontal gyrus, orbital part & $.050^{\mathrm{a}}$ & $.012^{\mathrm{a}}$ \\
L. Inferior frontal gyrus, opercular part & $.001^{\mathrm{b}}$ & $<.001^{\mathrm{b}}$ \\
L. Inferior frontal gyrus, triangular part & $.001^{\mathrm{b}}$ & $<.001^{\mathrm{b}}$ \\
L. Calcarine & $.029^{\mathrm{a}}$ & $.043^{\mathrm{a}}$ \\
L. Rolandic operculum & $.001^{\mathrm{b}}$ & $.001^{\mathrm{b}}$ \\
L. Insula & $.002^{\mathrm{b}}$ & $.001^{\mathrm{b}}$ \\
L. Anterior cingulate and paracingulate gyri & $.002^{\mathrm{b}}$ & $.001^{\mathrm{a}}$ \\
R. Anterior cingulate and paracingulate gyri & $.046^{\mathrm{a}}$ & $.005^{\mathrm{b}}$ \\
R. Median cingulate and paracingulate gyri & $.010^{\mathrm{a}}$ & $.006^{\mathrm{b}}$ \\
L. Amygdala & $.013^{\mathrm{a}}$ & $.013^{\mathrm{a}}$ \\
R. Cuneus & $.038^{\mathrm{a}}$ & $.031^{\mathrm{a}}$ \\
L. Postcentral gyrus & $.004^{\mathrm{b}}$ & $.044^{\mathrm{a}}$ \\
R. Lingual gyrus & $.035^{\mathrm{a}}$ & $.008^{\mathrm{b}}$ \\
R. Angular gyrus & $.023^{\mathrm{a}}$ & $.020^{\mathrm{a}}$ \\
R. Superior parietal gyrus & $.014^{\mathrm{a}}$ & $.015^{\mathrm{a}}$ \\
L. Supramarginal gyrus & $.001^{\mathrm{b}}$ & $.002^{\mathrm{b}}$ \\
L. Caudate & $.002^{\mathrm{b}}$ & $.006^{\mathrm{b}}$ \\
R. Caudate & $.048^{\mathrm{a}}$ & $.013^{\mathrm{a}}$ \\
L. Heschl gyrus & $.002^{\mathrm{b}}$ & $.001^{\mathrm{b}}$ \\
L. Superior temporal gyrus & $.001^{\mathrm{b}}$ & $.001^{\mathrm{b}}$ \\
R. Superior occipital gyrus & $.014^{\mathrm{a}}$ & $.023^{\mathrm{a}}$ \\
R. Middle occipital gyrus & $.006^{\mathrm{b}}$ & $.015^{\mathrm{a}}$ \\
R. Inferior occipital gyrus & $.024^{\mathrm{a}}$ & $.041^{\mathrm{a}}$ \\
\hline Not & &
\end{tabular}

Note:-L. indicates left; R., right.

a $P<.05$.

$\mathrm{b} p<.01$.

Nodal Characteristics. The degree of a node is the number of connections between other nodes and the node and reflects the interaction between the node and its neighbors. Nodal efficiency is the average of the inverse of the shortest path length between the node of interest and all other nodes, and it measures the ability of a node to transmit information to other nodes in the network. The betweenness centrality can quantify how central a node is located within a network and the role it plays in facilitating communication with other nodes.

Identification of Hubs. Brain regions with a large number of connections are considered hubs, characterized by their high degree of connectivity to other regions and small characteristic shortest path lengths. We used betweeness centrality and degree as the hub score for identifying the hubs of a network. All the mea- sures were averaged between 2 hemispheres. Regions were ranked by nodal betweeness centrality and degree. The top $20 \%$ of brain regions were assigned as network hubs.

\section{Statistical Analysis}

To determine the between-group difference in all the aforementioned network metrics of all 3 cohorts, we used 1-way ANOVA with 90 regional brain volumes and the absence or presence of lacunar infarcts, chronic infarcts, and microbleeds as covariates. Post hoc analyses were performed with corrections for multiple comparisons by using Bonferroni correction. A significance level of $P<.05$ was set for all statistical tests. The software package SPSS 22.0 (IBM, Armonk, New York) was used for all the statistical analyses.

\section{RESULTS \\ Demographics}

One patients with SLE and 1 with NPSLE were excluded due to imaging artifacts. There was no significant difference in age among all cohorts and no difference in disease duration, total cumulative prednisolone use (from disease onset until the time of study), and the prevalence of radiologic abnormalities of patients with NPSLE compared with those with SLE. All demographics, radiologic abnormalities, prevalence of various neuropsychiatric symptoms, and $P$ values of group comparisons are shown in Table 1.

\section{Volumetric Analysis}

There were widespread decreases in regional brain volumes for patients with both NPSLE and SLE compared with controls (Table 2). The differences between patients with NPSLE and those with SLE were not statistically significant.

\section{Small-Worldness}

The structural brain network of all cohorts was consistent with the small-world network $(\gamma / \lambda$ for NPSLE, $2.91 \pm 0.26 / 1.30 \pm$ 0.03 ; SLE, $3.01 \pm 0.28 / 1.29 \pm 0.03$; control, $2.97 \pm 0.38 / 1.28 \pm$ $0.03)$. Compared with controls, patients with NPSLE had a significantly decreased clustering coefficient $(P=.031)$ and increased characteristic shortest path length $(P<.001)$, whereas patients with SLE had significantly higher characteristic shortest path length $(P=.012)$. Compared with patients with SLE, patients with NPSLE had a significantly lower clustering coefficient $(P=.034)$. 
Table 3: Hubs identified from each cohort

\begin{tabular}{|c|c|c|c|c|}
\hline Regions & Abbreviation & Controls & NPSLE & SLE \\
\hline Precuneus & PCUN & $\sqrt{ }$ & $\sqrt{ }$ & $\sqrt{ }$ \\
\hline Insula & INS & $\sqrt{ }$ & $\sqrt{ }$ & $\sqrt{ }$ \\
\hline Putamen & PUT & $\checkmark$ & $\sqrt{ }$ & $\sqrt{ }$ \\
\hline Hippocampus & HIP & $\sqrt{ }$ & $\sqrt{ }$ & $\sqrt{ }$ \\
\hline Caudate nucleus & CAU & $\sqrt{ }$ & $\sqrt{ }$ & $\sqrt{ }$ \\
\hline Superior frontal gyrus, medial & SFGmed & $\checkmark$ & $\sqrt{ }$ & $\sqrt{ }$ \\
\hline Thalamus & THA & $\sqrt{ }$ & & \\
\hline Median cingulate and paracingulate gyri & DCG & $\sqrt{ }$ & & \\
\hline Pallidum & PAL & $\sqrt{ }$ & & $\sqrt{ }$ \\
\hline Superior temporal gyrus & STG & & $\sqrt{ }$ & \\
\hline Lingual gyrus & LING & & $\sqrt{ }$ & \\
\hline Middle temporal gyrus & MTG & & $\sqrt{ }$ & $\sqrt{ }$ \\
\hline Temporal pole: middle temporal gyrus & TPOmid & & & $\sqrt{ }$ \\
\hline
\end{tabular}

Note:- $\sqrt{ }$ indicates identified as hub.
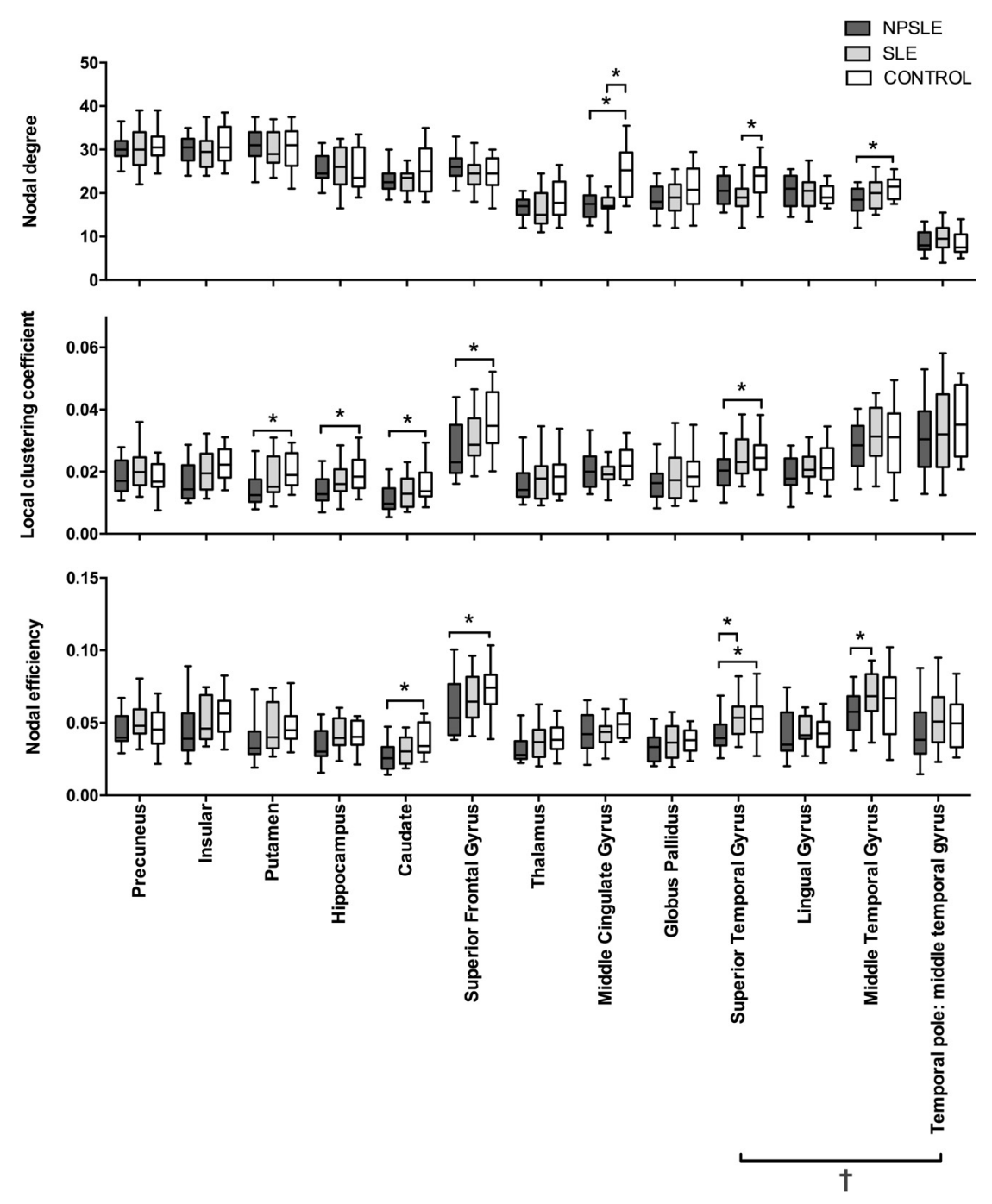

FIG 2. Boxplots showing the group comparisons (median, 50th percentile values, maximum, and minimum) for nodal degree, local clustering coefficient, and nodal efficiency of the 9 network hubs identified from healthy controls and 4 identified from patients with SLE or NPSLE. The nodal degree of the middle cingulate cortex $(P=.005)$ was decreased for both patients with NPSLE and those with SLE compared with controls. For patients with NPSLE, the local clustering coefficients of the superior frontal cortex $(P=.003)$, hippocampus $(P=.027)$, caudate $(P=.018)$, and putamen $(P=.010)$ were decreased. The nodal efficiency of the superior frontal cortex $(P=.048)$ and caudate $(P=.004)$ were also decreased. Compared with patients with SLE, patients with NPSLE had significantly lower nodal efficiency in the superior temporal gyrus $(P=.046)$ and middle temporal gyrus $(P=.041)$. The asterisk indicates $P<.05$; dagger, network hubs identified from patients with SLE or NPSLE.

\section{Network Efficiency}

Compared with controls, only patients with NPSLE had significantly lower global efficiency $(P=.010)$ and local efficiency $(P=.035)$. Compared with patients with SLE, patients with NPSLE had significantly lower local efficiency $(P=.049)$.

\section{Nodal Characteristics}

From each cohort, 9 of 45 cortical and subcortical regions in each hemisphere were identified as hubs according to the descending order of hub scores (Table $3)$. Contrary to the controls, the following brain regions were identified as hubs in the NPSLE cohort: the lingual, superior temporal, and middle temporal gyri; and in the SLE cohort: the middle temporal gyrus. The nodal characteristics of the hubs identified from all cohorts were compared and are shown in Fig 2. Compared with controls, patients with SLE $(P=.004)$ and NPSLE $(P=$ $.005)$ had a significantly decreased nodal degree in the middle cingulate cortex. The local clustering coefficients of the superior frontal cortex $(P=.003)$, hippocampus $(P=.027)$, caudate $(P=.018)$, and putamen $(P=.010)$ and the nodal efficiency of the superior frontal cortex $(P=.048)$ and caudate $(P=.004)$ of patients with NPSLE were also significantly reduced. Compared with patients with SLE, patients with NPSLE had significantly lower nodal efficiency in the superior temporal gyrus $(P=$ $.046)$ and middle temporal gyrus $(P=$ $.041)$.

\section{DISCUSSION}

The coordination and dynamic interactions among different brain regions are critical to normal cognition and behavior. ${ }^{24}$ Disruption of this complex brain network due to neuropsychiatric disorders, such as Alzheimer disease ${ }^{25}$ or schizophrenia, ${ }^{26}$ could lead to cognitive and behavioral deficits in addition to local neurologic sequelae. ${ }^{7}$

In the current study, we have demonstrated that there are more extensive local and global alterations in the structural brain network of patients with NPSLE compared with those with SLE and that the findings thereof were independent of regional brain volumetric changes and hence were likely micro- 
structural in nature. In a similar vein, several studies ${ }^{6,27,28}$ have observed a larger extent of focal microstructural alterations in patients with NPSLE than in those with SLE. For instance, Jung et $\mathrm{al}^{6}$ demonstrated significant changes in the fractional anisotropy and mean diffusivity of the body of the corpus callosum, left forceps major, and left anterior corona radiata in patients with NPSLE, but not in patients with SLE, compared with controls. Zimny et $\mathrm{al}^{28}$ have shown that the fractional anisotropy of the left inferior longitudinal and inferior fronto-occipital fasciculi of patients with NPSLE was lower compared with patients with SLE.

Together, these findings lead us to believe that there is a likely association between neuropsychiatric symptoms and the extent of alterations in the structural brain network of patients with NPSLE.

\section{Global Network Reorganization}

Our results show that the structural brain network of both patient cohorts was consistent with an intact small-world network, albeit a decrease in the clustering coefficient of patients with NPSLE and an increase in the characteristic shortest path length of both patient cohorts compared with controls. The fact that the structural brain network of patients with NPSLE had a lower clustering coefficient compared with patients with SLE suggests that the balance between functional segregation and integration for patients with NPSLE may be compromised. ${ }^{7}$ Thus, their functional brain network may have to reconfigure to meet and compensate for the demand necessary for maintaining the working capacity of the structural brain network in effortful cognitive tasks. ${ }^{29}$

From the perspective of network efficiency, only the structural brain network of patients with NPSLE had lower global and local efficiencies compared with controls and lower local efficiency than patients with SLE. A decrease in the global efficiency of a brain network suggests a loss of long-range connections, likely as a result of compromised cognitive capacity. ${ }^{30}$ On the other hand, a decrease in the local efficiency suggests a loss of short-range connections throughout the entire brain network, likely attributable to changes in the modularized information-processing ability and fault tolerance in information transfer of the network. ${ }^{30}$

\section{Local Network Reorganization}

Of the 9 brain regions identified as hubs from controls, the thalamus, middle cingulate cortex, and globus pallidus were replaced by lingual, superior temporal, and middle temporal gyri for patients with NPSLE (Fig 2). That the former 3 brain regions were no longer hubs is largely consistent with the behavioral and cognitive deficits that these patients often endure. ${ }^{31-33}$ On the other hand, the thalamus and middle cingulate cortex were replaced by the middle temporal gyrus for patients with SLE. As is evident from Fig 2, there are more extensive changes in the nodal characteristics of various brain hubs of patients with NPSLE than in those with SLE compared with controls. These results indicate that the role of these hubs was weakened, ${ }^{10}$ likely related to the cognitive deficits and emotional instability commonly seen in patients with NPSLE. ${ }^{34}$

\section{Brain Network Alterations and Neuropsychiatric Manifestations in SLE}

The findings from the current study indicate that there were more extensive local and global reorganizations in the structural brain network of patients with NPSLE than in those with SLE and that these changes are the likely attempts of the network to compensate for the neuropsychiatric symptoms commonly seen in the patients with NPSLE. Apart from the structural network, reorganization of the functional brain network of these patients has also been observed in functionally similar brain regions. ${ }^{33,35-38}$ For instance, Fitzgibbon et $\mathrm{al}^{33}$ demonstrated that patients with NPSLE had larger functional MR imaging activations during working memory tasks compared with controls. Rocca et $\mathrm{al}^{36}$ showed that patients with NPSLE had altered cortical fMRI activation during simple motor tasks. Mak et $\mathrm{al}^{35}$ have shown that additional cortical regions were recruited for the executive function of patients with SLE. ${ }^{35}$ Differential fMRI activations for the visuoconstructional ability, working memory, and attention of children with childhood-onset SLE were also observed by DiFrancesco et al. ${ }^{37}$ These functional network reorganizations further supported the notion of increased recruitment of extra cortical pathways to compensate for the cognitive or behavioral deficits of these patients.

\section{CONCLUSIONS}

We have demonstrated that there were more extensive local and global alterations in the structural brain network of patients with NPSLE than in those with SLE. Brain connectivity analysis may be a diagnostic tool to subtype these patients, thereby potentially allowing the appropriate therapeutics to be arranged, especially when a more aggressive treatment regimen is necessary for those with NPSLE.

Disclosures: Mo Yin Mok_UNRELATED: Grants/Grants Pending: General Research Fund: Comments: fund in Hong Kong for a basic science project; Payment for Lectures (including service on Speakers Bureaus): Roche, Pfizer, and Janssen; Comments: honorarium given as speakers for isolated educational events organized by pharmaceutical companies. Wallace Chak Sing Lau-UNRELATED: Consultancy: Pfizer (as advisor to their Asia Rheumatology Expert Advisory Council for Health program); Employment: the University of Hong Kong (as clinical professor of medicine); Grants/Grants Pending: General Research Fund, Hong Kong; Health and Medical Research Fund, Hong Kong; Payment for Lectures (including service on Speakers Bureaus): honorarium given as speaker for Asia Rheumatology Expert Advisory Council for Health.

\section{REFERENCES}

1. Jeltsch-David H, Muller S. Neuropsychiatric systemic lupus erythematosus: pathogenesis and biomarkers. Nat Rev Neurol 2014; 10:579-96 CrossRef Medline

2. Hanly JG. Diagnosis and management of neuropsychiatric SLE. Nat Rev Rheumatol 2014;10:338-47 CrossRef Medline

3. Luyendijk J, Steens SCA, Ouwendijk WJ, et al. Neuropsychiatric systemic lupus erythematosus: lessons learned from magnetic resonance imaging. Arthritis Rheum 2011;63:722-32 CrossRef Medline

4. Bosma GPT, Middelkoop HA, Rood MJ, et al. Association of global brain damage and clinical functioning in neuropsychiatric systemic lupus erythematosus. Arthritis Rheum 2002;46:2665-72 CrossRef Medline

5. Lee SP, Wu CS, Hsieh LC, et al. Efficacy of magnetic resonance diffusion tensor imaging and three-dimensional fiber tractography in the detection of clinical manifestations of central nervous system lupus. Magn Reson Imaging 2014;32:598-603 CrossRef Medline 
6. Jung RE, Caprihan A, Chavez RS, et al. Diffusion tensor imaging in neuropsychiatric systemic lupus erythematosus. BMC Neurol 2010; 10:65 CrossRef Medline

7. Bullmore $E$, Sporns $O$. The economy of brain network organization. Nat Rev Neurosci 2012;13:336-49 CrossRef Medline

8. Gratton C, Nomura EM, Pérez F, et al. Focal brain lesions to critical locations cause widespread disruption of the modular organization of the brain. J Cogn Neurosci 2012;24:1275-85 CrossRef Medline

9. Bullmore E, Sporns O. Complex brain networks: graph theoretical analysis of structural and functional systems. Nat Rev Neurosci 2009;10:186-98 CrossRef Medline

10. van den Heuvel MP, Sporns O. Network hubs in the human brain. Trends Cogn Sci 2013;17:683-96 CrossRef Medline

11. Shi F, Wang L, Peng Z, et al. Altered modular organization of structural cortical networks in children with autism. PLoS One 2013;8: e63131 CrossRef Medline

12. de Haan W, Mott K, van Straaten EC, et al. Activity dependent degeneration explains hub vulnerability in Alzheimer's disease. PLoS Comput Biol 2012;8:e1002582 CrossRef Medline

13. Hochberg MC. Updating the American College of Rheumatology revised criteria for the classification of systemic lupus erythematosus. Arthritis Rheum 1997;40:1725-25 CrossRef Medline

14. The American College of Rheumatology nomenclature and case definitions for neuropsychiatric lupus syndromes. Arthritis Rheum 1999;42:599-608 Medline

15. Fischl B, Salat DH, Busa E, et al. Whole brain segmentation: automated labeling of neuroanatomical structures in the human brain. Neuron 2002;33:341-55 CrossRef Medline

16. Smith SM, Jenkinson M, Woolrich MW, et al. Advances in functional and structural MR image analysis and implementation as FSL. Neuroimage 2004;23(suppl 1):S208-19 CrossRef Medline

17. Brown JA, Rudie JD, Bandrowski A, et al. The UCLA multimodal connectivity database: a web-based platform for brain connectivity matrix sharing and analysis. Front Neuroinform 2012;6:28 CrossRef Medline

18. Woolrich MW, Jbabdi S, Patenaude B, et al. Bayesian analysis of neuroimaging data in FSL. Neuroimage 2009;45:S173-86 CrossRef Medline

19. Wang R, Benner T. Diffusion toolkit: a software package for diffusion imaging data processing and tractography. In: Proceedings of the European Society for Magnetic Resonance in Medicine and Biology and the International Society for Magnetic Resonance in Medicine Joint Annual Meeting. Berlin, Germany; May 19-25, 2007;15:3720

20. Rubinov M, Sporns O. Complex network measures of brain connectivity: uses and interpretations. Neuroimage 2010;52: 1059-69 CrossRef Medline

21. Watts DJ, Strogatz SH. Collective dynamics of "small-world" networks. Nature 1998;393:440-42 CrossRef Medline

22. Latora V, Marchiori M. Economic small-world behavior in weighted networks. Eur Phys J B 2003;32:249-63 CrossRef
23. Latora V, Marchiori M. Efficient behavior of small-world networks Phys Rev Lett 2001;87:198701 CrossRef Medline

24. Pessoa L. On the relationship between emotion and cognition. Nat Rev Neurosci 2008;9:148-58 CrossRef Medline

25. Lo CC, Wang PP, Chou KH, et al. Diffusion tensor tractography reveals abnormal topological organization in structural cortical networks in Alzheimer's disease. J Neurosci 2010;30:16876-85 CrossRef Medline

26. Wang Q, Su T-P, Zhou Y, et al. Anatomical insights into disrupted small-world networks in schizophrenia. Neuroimage 2012;59: 1085-93 CrossRef Medline

27. Schmidt-Wilcke T, Cagnoli P, Wang P, et al. Diminished white matter integrity in patients with systemic lupus erythematosus. Neuroimage Clin 2014;5:291-97 CrossRef Medline

28. Zimny A, Szmyrka-Kaczmarek M, Szewczyk P, et al. In vivo evaluation of brain damage in the course of systemic lupus erythematosus using magnetic resonance spectroscopy, perfusionweighted and diffusion-tensor imaging. Lupus 2014;23:10-19 CrossRef Medline

29. Bassett DS, Wymbs NF, Porter MA, et al. Dynamic reconfiguration of human brain networks during learning. Proc Natl Acad Sci U S A 2011;108:7641-46 CrossRef Medline

30. Achard S, Bullmore E. Efficiency and cost of economical brain functional networks. PLoS Comput Biol 2007;3:e17 CrossRef Medline

31. Mittal M, Khan S. Starvation causes acute psychosis due to anterior thalamic infarction. South Med J 2010;103:701-03 CrossRef Medline

32. Ren T, Ho RC-M, Mak A. Dysfunctional cortico-basal ganglia-thalamic circuit and altered hippocampal-amygdala activity on cognitive set-shifting in non-neuropsychiatric systemic lupus erythematosus. Arthritis Rheum 2012;64:4048-59 CrossRef Medline

33. Fitzgibbon BM, Fairhall SL, Kirk IJ, et al. Functional MRI in NPSLE patients reveals increased parietal and frontal brain activation during a working memory task compared with controls. Rheumatology 2008;47:50-53 CrossRef Medline

34. Goldin PR, McRae K, Ramel W, et al. The neural bases of emotion regulation: reappraisal and suppression of negative emotion. Biol Psychiatry 2008;63:577-86 CrossRef Medline

35. Mak A, Ren T, Fu EH, et al. A prospective functional MRI study for executive function in patients with systemic lupus erythematosus without neuropsychiatric symptoms. Semin Arthritis Rheum 2012; 41:849-58 CrossRef Medline

36. Rocca MA, Agosta F, Mezzapesa DM, et al. An fMRI study of the motor system in patients with neuropsychiatric systemic lupus erythematosus. Neuroimage 2006;30:478-84 CrossRef Medline

37. DiFrancesco MW, Gitelman DR, Klein-Gitelman MS, et al. Functional neuronal network activity differs with cognitive dysfunction in childhood-onset systemic lupus erythematosus. Arthritis Res Ther 2013;15:R40 CrossRef Medline

38. Mikdashi JA. Altered functional neuronal activity in neuropsychiatric lupus: a systematic review of the fMRI investigations. Semin Arthritis Rheum 2016;45:455-62 CrossRef Medline 